\title{
A novel airway management technique for nasal surgery following transoral robotic surgery for patients with obstructive sleep apnea
}

\author{
Jerry Tan, MBBS • Priscilla Phoon, MBBS • Theodore Wong, MD • \\ Ruban Poopalalingam, MBBS
}

Received: 24 July 2015/Revised: 17 August 2015/Accepted: 26 August 2015/Published online: 29 August 2015

(C) Canadian Anesthesiologists' Society 2015

\section{To the Editor,}

Multilevel surgical intervention to treat obstructive sleep apnea (OSA) has been shown to result in a significant improvement in OSA symptoms. This approach involves resection of pertinent anatomical obstructions to airflow, such as the hypopharynx, base of the tongue, or the epiglottis, with or without uvulopalatopharyngoplasty. ${ }^{1}$ In addition, correction of nasal obstruction in OSA patients has been recommended independent of OSA severity as it has been found to improve subjective snoring and daytime fatigue. $^{2}$

In our institution, drug-induced sleep endoscopy is performed before commencing surgery to confirm the pattern of airway obstruction. ${ }^{3}$ Subsequently, anesthesia is induced and nasal intubation is performed for transoral robotic surgery (TORS) with a plan for oral reintubation in case nasal surgery is required.

Patients with OSA are more likely to have a difficult airway. ${ }^{4}$ The requirement to reintubate these patients for the second-stage nasal procedure is made further difficult (or potentially impossible) by airway edema and bleeding following TORS. It can also make bag and mask ventilation difficult. Furthermore, reintubation attempts can cause trauma to the nasal or oropharyngeal structures as well as increase the risk of aspiration of blood and debris. More invasive alternatives, such as tracheostomy or submental intubation, ${ }^{5}$ can be considered in extreme cases, but these options are undesirable in the elective setting.

J. Tan, MBBS $(\bowtie) \cdot$ P. Phoon, MBBS · T. Wong, MD .

R. Poopalalingam, MBBS

Department of Anesthesiology, Singapore General Hospital,

Singapore, Singapore

e-mail: jerry.tan@mohh.com.sg
To overcome these problems, we have devised an alternative approach aimed at avoiding extubation. In our institution, we use the Ivory PVC, North Facing, Nasal, Profile Soft Seal Cuff, Polar Preformed Endotracheal Tube (Smiths Medical International Ltd; http://www.smithsmedical.com) for nasal intubation. Upon completion of TORS, we suction the oral cavity and check the cuff pressure of the endotracheal tube (ETT) to ensure no leakage in the ventilation system. We next tie a knot in the pilot balloon tube close to the ETT, cut off the pilot balloon, disconnect the Ivory tube from the circuit, and remove its $15-\mathrm{mm}$ connector. With an assistant's help, we cut off and lubricate the proximal $5-8 \mathrm{~cm}$ of the ETT, and then push the ETT into the patient's nasal cavity. To facilitate pushing the proximal end of the Ivory tube into the nasal cavity, a second tube of the same size can be used to apply a steady downward pressure while under direct laryngoscopy. The Ivory tube can be simultaneously "fished" out from the oral cavity with Magill forceps and then re-connected to the ventilator. An alternate method to minimize inadvertent extubation is to use the laryngoscope blade to compress the distal portion of the ETT against the posterior pharynx as the proximal portion is "fished" out. Its position should be confirmed with capnography and checked clinically for bilaterally equal air entry before securing the ETT in its new position. Subsequently, the nasal surgery can proceed. At the end of the nasal procedure, we cut off the pilot tube knot to allow egress of air from the ETT cuff before extubation (Figure).

It is important to oxygenate the patient's lungs adequately and to have measures in place for reintubation in case of accidental extubation while withdrawing the proximal end of the ETT from the oral cavity. A mouth pack can be inserted if there is significant cuff leakage after ETT adjustment. Importantly, at the time of subsequent 

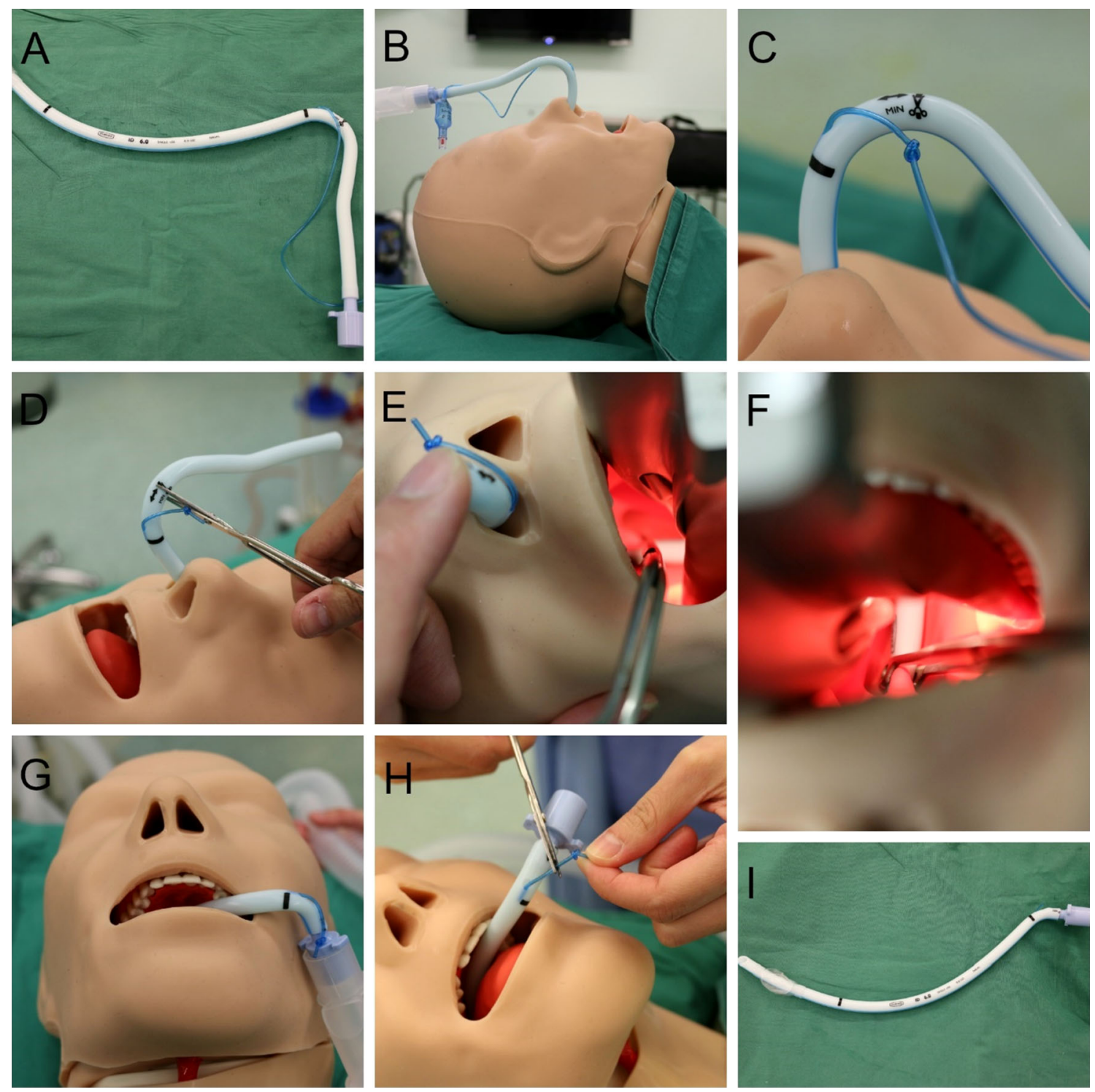

Figure A) Appearance of Ivory tube pre-intubation. B) Position of Ivory PVC, Nasal, Profile Soft Seal cuff tracheal tube (Smiths Medical) for transoral robotic surgery. C) A knot tied on the pilot tube as proximal to the Ivory tube as possible. D) Ivory tube cut after disconnecting the $15-\mathrm{mm}$ standard connector and ventilation circuit. E) Downward pressure of the Ivory tube into the nasal cavity and

extubation, the ETT should be removed slowly to allow sufficient air to egress in order to avoid injuring the vocal cords.

Surgical strategies for OSA are evolving, and multilevel surgery in a single setting is becoming increasingly common in an attempt to avoid repeated anesthetics. An alternative method of ETT exchange should be considered in OSA patients who are already at high risk of airway complications.

Funding No external funding. direct laryngoscopy. F) Pharyngeal view showing the use of Magill forceps to "fish" out the proximal end of the nasal tube. G) Final position of endotracheal tube for nasal surgery. H) Cutting the knot from the pilot tube to allow egress of air for extubation. I) Appearance of the Ivory tube post extubation

Competing interests No competing interests declared.

\section{References}

1. Thaler ER, Rassekh CH, Lee JM, Weinstein GS, O'Malley BW Jr. Outcomes for multilevel surgery for sleep apnea: obstructive sleep apnea, transoral robotic surgery, and uvulopalatopharyngoplasty. Laryngoscope 2015; . DOI:10.1002/lary.25353.

2. Rombaux $P$, Liistro $G$, Hamoir $M$, et al. Nasal obstruction and its impact on sleep-related breathing disorders. Rhinology 2005; 43 : 242-50. 
3. Mathews AM, Goh JP, Teo LM. A case report on the anaesthetic management of dexmedetomidine-induced sleep endoscopy and transoral robotic surgery for the treatment of obstructive sleep apnoea. Proceedings of Singapore Healthcare 2013; 22: 151-5.

4. Stierer TL. Obstructive sleep apnea, sleep disorders, and perioperative considerations. Anesthesiol Clin 2015; 33: 305-14.
5. Caubi AF, Vasconcelos BC, Vasconcellos RJ, de Morais HH, Rocha NS. Submental intubation in oral maxillofacial surgery: review of the literature and analysis of 13 cases. Med Oral Patol Oral Cir Bucal 2008; 13: E197-200. 